Check for updates

Cite this: RSC Adv., 2019, 9, 17358

Received 24th February 2019

Accepted 27th May 2019

DOI: $10.1039 / c 9 r a 01394 f$

rsc.li/rsc-advances

\section{Polyaniline nanorods random assembly on sugarcane bagasse pith-based carbon sheets with promising capacitive performance $\dagger$}

\author{
Kezheng Gao, Shuyan Zhao, Qingyuan Niu (D) and Lizhen Wang*
}

Polyaniline (PANI) nanorods were randomly deposited on oxidized 2D sugarcane pith-based porous carbon nanosheets by using dilute polymerization methods. The random stacked morphology of the PANI nanorods on the oxidized pith-based porous carbon nanosheets (SPCN) can be effectively controlled by simply changing the molar mass of aniline monomer. When the molar mass of the aniline monomer is increased to $0.02 \mathrm{M}$, the PANI nanorods can be randomly and uniformly stacked on the oxidized SPCN. Most of these stacked pores derived from random stack of the PANI nanorods on the oxidized SPCN are mesopore and macropore. These stacked pores not only facilitate the diffusion of ions in the stacked layer of the PANI nanorods, but also mitigate mechanical deformation of the PANI nanorods during the doping/dedoping process. Furthermore, the relationship between the properties of the oxidized SPCN/ PANI- $X$ ( $X$ represents the molar mass of aniline monomer) electrode materials and molar mass of aniline monomer is explored in detail. The oxidized SPCN/PANI-0.02 exhibits the best electrochemical performance in $1 \mathrm{M} \mathrm{H}_{2} \mathrm{SO}_{4}$. The largest specific electrode capacitance is up to $513 \mathrm{~F} \mathrm{~g}^{-1}$ at a current density of $0.25 \mathrm{~A} \mathrm{~g}^{-1}$. The oxidized SPCN/PANI- 0.02 also exhibits excellent electrochemical cycling stability.

\section{Introduction}

Polyaniline (PANI), as an electronically conductive polymer, has received extensive attention in the field of energy storage due to its high theoretical specific capacitance (originating from its multiple redox states). ${ }^{1-4}$ Usually, the insertion/expulsion of dopants (counter-anions) mainly takes place in the surface layer of PANI bulk materials during charge/discharge process. ${ }^{5,6}$ Therefore, the surface layer of PANI materials has the largest contribution to the capacitance. In addition, the mechanical deformation of PANI electrode materials will inevitably occur during insertion/expulsion of dopants process, which can impart poor electrochemical performance of polyaniline (especially cyclic stability). ${ }^{7,8}$ Therefore, in order to fully exploit the potential of PANI for a very high specific capacitance, the PANI electrode materials should not only be a nanomaterial (the high surface area available for charging), but also should be able to form specific aggregated forms (mitigates mechanical deformation). ${ }^{9-12}$

Among the PANI nanomaterials, the PANI nanorods (or nanofibers) have attracted widespread attention due to their easy synthesis, solvent processability and controllable unique

School of Material and Chemical Engineering, Zhengzhou University of Light Industry, Zhengzhou 450002, P. R. China. E-mail: niuqingyuan1984@126.com; wlz@zzuli.edu. cn

$\dagger$ Electronic supplementary information (ESI) available: Fig. S1-S4, Tables S1-S3. See DOI: 10.1039/c9ra01394f aggregation morphology. ${ }^{13,14}$ However, the poor electrochemical stability and the cycle stability limits their widespread application in supercapacitors. In recent years, many recent researches have focused on combining PANI nanomaterials with carbon nanomaterials (the carbon materials can be 1D nanofiber, 2D nanosheet, and 3D network) due to the excellent conductivity and electrochemical stability of carbon materials. ${ }^{15-20}$ The carbon nanomaterial can significantly improve the cycle stability and rate capability of the composite electrode material, while the PANI nanorods will significantly increase the specific capacitance of the composite electrode material. The PANI nanorods can exhibit an array morphology which vertically aligned on carbon nanomaterials. Such composite electrode materials can exhibit good electrochemical performance due to their optimal pathway for ion diffusion. ${ }^{21,22}$ In addition to the array morphology, the random stacked morphology is another important morphology. A large number of stacked hierarchical pore structures will be formed during the random stacking of the PANI nanorods. The size of these stacked pores is depending on the morphology and size of the PANI nanorods. These stacked pores will not only facilitate the diffusion of ions, but also mitigates mechanical deformation of the PANI nanorods during the doping/dedoping process. Therefore, the random stacked morphology of the PANI nanorods will also have excellent electrochemical performance in my opinion.

The biomass-based porous carbon materials have drawn much attention due to their extensive sources and environmental friendliness in recent years. 1D, 2D and 3D biomass- 
based porous carbon materials have been successfully prepared so far. ${ }^{23-28}$ Among the different microstructures of the biomassbased carbon materials, two-dimensional (2D) porous carbon nanosheets may be more suitable for preparing random stacked PANI nanorods composite electrode materials due to their high aspect ratios. Sugarcane pith is an effective natural precursor material for the large-size $2 \mathrm{D}$ porous carbon nanosheets due to their abundant parenchyma cell walls. ${ }^{29}$ 2D sugarcane pithbased porous carbon nanosheets (SPCN) may be one of the most suitable 2D carbon nanosheet materials for the random stacked PANI nanorods from the perspective of environmental protection, economy and sustainable development.

In this article, sugarcane pith was selected as the selftemplate of $2 \mathrm{D}$ carbon nanosheets. And then the PANI nanorods were randomly deposited on the oxidized 2D sugarcane pith-based porous carbon nanosheets by using the dilute polymerization methods. The different molar masses of aniline monomer were used to study the effect on the random stacked morphology and electrochemical performance of the oxidized SPCN/PANI- $X$ electrode materials.

\section{Experimental}

\section{The preparation of oxidized SPCN}

Sugarcane piths were collected from a supermarket. In order to remove the residual sucrose, the sugarcane bagasse piths were repeated rinsed with distilled water for about one week. After drying ( $60{ }^{\circ} \mathrm{C}$ for 10 hours), smashing and screening, most of the screened sugarcane piths present a sheet-like morphology. The screened sugarcane piths were put into a tube furnace and carbonized under a flowing Ar atmosphere at $700{ }^{\circ} \mathrm{C}$ with a ramping rate of $3{ }^{\circ} \mathrm{C} \mathrm{min}^{-1}$ for $3 \mathrm{~h}$ to obtain SPCN. SPCN $(0.2$ g) was added to concentrated sulfuric acid $\left(\mathrm{H}_{2} \mathrm{SO}_{4}, 48 \mathrm{~mL}\right)$ and stirred for 1 hour. After adding phosphoric acid $\left(\mathrm{H}_{3} \mathrm{PO}_{4}\right.$, about 5 $\mathrm{mL}$ ) for stirring 15 minutes, $\mathrm{KMnO}_{4}(1 \mathrm{~g})$ was added slowly and reacted at $65{ }^{\circ} \mathrm{C}$ for 2 hours. After cooling to room temperature, the mixture was poured into water (about $4{ }^{\circ} \mathrm{C}, 400 \mathrm{~mL}$ ), then add $20 \mathrm{~mL} \mathrm{H}_{2} \mathrm{O}_{2}$. The solution was allowed to stand overnight and then rinsed with water until neutral. The oxidized SPCN were obtained after drying.

\section{The preparation of oxidized SPCN/PANI composite materials}

Oxidized SPCN/PANI composite materials were synthesized by dilute polymerization. The oxidized SPCN $(0.050 \mathrm{~g})$ was added to the mixed solution of $1 \mathrm{M} \mathrm{HClO}_{4}(75 \mathrm{~mL})$ and ethanol $(25 \mathrm{~mL})$ and then stored at $4{ }^{\circ} \mathrm{C}$ for 10 hours. Aniline monomer (227.8 $\mu \mathrm{L}$ ) was added into the mixed solution and stirred for about $5 \mathrm{~min}$ to form a uniform mixture. And then the above mixture was added to $25 \mathrm{~mL} 1 \mathrm{M} \mathrm{HClO}_{4}$ (about $4{ }^{\circ} \mathrm{C}$ ) containing $0.382 \mathrm{~g}$ $\left(\mathrm{NH}_{4}\right)_{2} \mathrm{~S}_{2} \mathrm{O}_{8}$ (APS, the molar concentration of aniline in the reaction system is $0.02 \mathrm{M}$ ). The polymerization reaction was continued for 16 hours at $4{ }^{\circ} \mathrm{C}$ under continuous stirring. Repeatedly washed with deionized water and ethanol, and the oxidized SPCN/PANI-0.02 composite materials were obtained after drying. The oxidized SPCN/PANI- $X(X$-molar concentration of aniline monomer, 0.005 M, 0.01 M, and 0.04 M) composite materials were prepared under the same conditions.

\section{Supercapacitors preparation and electrochemical measurements}

The electrochemical performances of the oxidized SPCN/PANI- $X$ were evaluated in a three-electrode and symmetrical twoelectrode system. The electrode materials were prepared by mixing oxidized SPCN/PANI- $X$ (80 wt\%), acetylene black (10 wt $\%$ ), and PTFE binder (10 wt $\%$ ) in ethanol. The obtained electrode materials were coated onto the $1 \times 1 \mathrm{~cm}$ stainless steel mesh (about $5 \mathrm{mg} \mathrm{cm} \mathrm{cm}^{-2}$ ). The prefabricated electrodes were dried at $80{ }^{\circ} \mathrm{C}$ for $12 \mathrm{~h}$ in a vacuum oven. After drying, the electrodes were pressed under a pressure of $10 \mathrm{MPa}$ for $1 \mathrm{~min}$. In the three electrode system, filter paper, saturated calomel electrode (SCE), activated carbon and $1 \mathrm{M} \mathrm{H}_{2} \mathrm{SO}_{4}$ were used as the separator, reference electrode, counter electrode and electrolyte, respectively. The potential window was chosen in the range of -0.2 to $0.8 \mathrm{~V}$ versus SCE. Two symmetrical oxidized SPCN/PANI-0.02 electrodes were separated by filter paper soaked with $1 \mathrm{M} \mathrm{H}_{2} \mathrm{SO}_{4}$. The electrochemical properties of the oxidized SPCN/PANI- $X$ were studied on a CHI 660E electrochemical workstation. The cut off charge voltage was set at 0$0.8 \mathrm{~V}$. Electrochemical impedance spectroscopy (EIS) tests were carried out in the frequency range of $105-0.001 \mathrm{~Hz}$ at a $5 \mathrm{mV}$ amplitude referring to open circuit potential.

\section{Material characterization}

The morphology evolution of the oxidized SPCN/PANI- $X$ was observed by Field-Emission Scanning Electron Microscope (FESEM). The structure of the oxidized SPCN/PANI- $X$ were measured by X-ray diffraction (XRD) with $\mathrm{Cu} \mathrm{K} \alpha 1$ radiation $(k=$ $0.15406 \mathrm{~nm}$ ) at $40 \mathrm{kV}$ and $30 \mathrm{~mA}$. Raman spectra were recorded on a HORIBA Scientific LabRAM HR Evolution Raman spectrometer system with an excitation wavelength of $532 \mathrm{~nm}$. The surface elemental characterization was performed by X-ray photoelectron spectroscopy (XPS) with a monochromatic Al $\mathrm{K} \alpha$ source. The content of specific elements in the sample was obtained by element analyzer. The specific surface area and pore size distribution of the samples were characterized by $\mathrm{N}_{2}$ adsorption analyses at $77 \mathrm{~K}$. The samples were outgassed at $150{ }^{\circ} \mathrm{C}$ for $12 \mathrm{~h}$ under a vacuum. The Barrett-Emmett-Teller (BET) method was used to evaluate specific surface area. The pore size distribution was calculated by using the NLDFT model.

\section{Results and discussion}

The morphology of SPCN and oxidized SPCN was characterized by FE-SEM observations (Fig. 1a and b). It can be clearly seen that SPCN exhibits a 2D sheet structure, which inherited from the microstructure of the precursor (Fig. S1 $\dagger$ ). After oxidation, oxidized SPCN still maintains the original 2D sheet structure. However, the size of oxidized SPCN becomes significantly smaller. The surface elemental composition and chemical state of the SPCN and oxidized SPCN were investigated by X-ray 

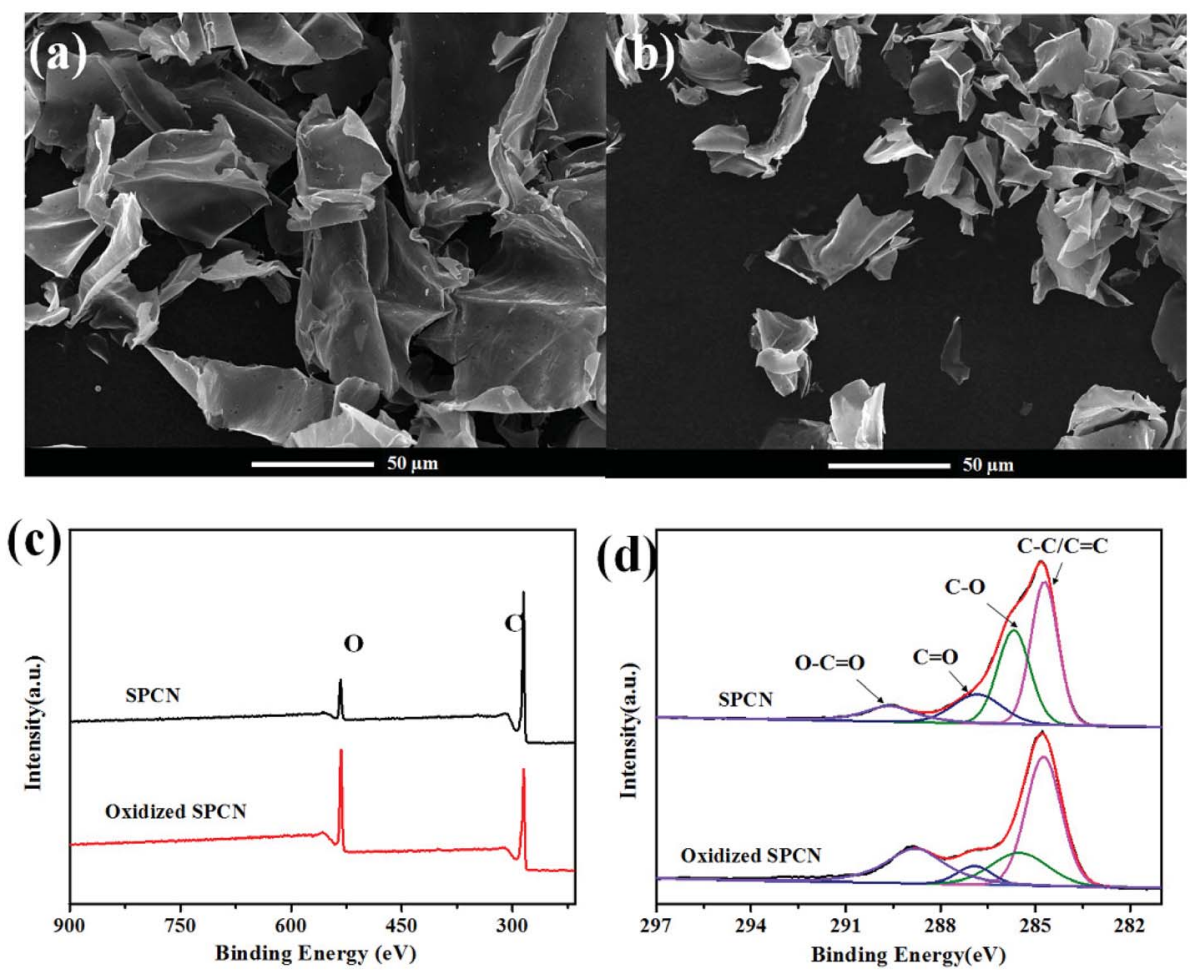

Fig. 1 FE-SEM of SPCN (a) and oxidized SPCN (b); XPS survey spectrum (c) of the SPCN and oxidized SPCN; high resolution C 1s XPS spectra (d) of the SPCN and oxidized SPCN.

photoelectron spectroscopy (XPS) analyses (Fig. 1c and d). The full scan spectrums of the SPCN and oxidized SPCN exhibit two peaks at around 284.8 and $532.1 \mathrm{eV}$ corresponding to the $\mathrm{C} 1 \mathrm{~s}$ and $\mathrm{O} 1 \mathrm{~s}$ peaks, respectively. Elemental analysis by XPS reveals that the surface oxygen content increased from $10.7 \%$ to $27.3 \%$ after oxidation treatment. The high-resolution $\mathrm{C} 1 \mathrm{~s}$ spectrums of the SPCN and oxidized SPCN can be deconvoluted into four peaks: the peaks at $284.7,285.6,286.8$, and $289.6 \mathrm{eV}$ are assigned to $\mathrm{C}-\mathrm{C} / \mathrm{C}=\mathrm{C}, \mathrm{C}-\mathrm{O}, \mathrm{C}=\mathrm{O}$, and $\mathrm{O}-\mathrm{C}=\mathrm{O}$, respectively. The relative percentages of carbon species are given in Table $\mathrm{S} 1 . \dagger$ Obviously, carboxyl carbon $(\mathrm{O}-\mathrm{C}=\mathrm{O})$ content significantly increased after oxidation treatment. These indicate that the hydrophilicity of the oxidized SPCN is significantly improved. This would be very beneficial for the uniform nucleation of the PANI nanorods on the oxidized SPCN during dilute polymerization.

The morphology evolution of the oxidized SPCN/PANI- $X$ with the increase of the PANI content is shown in Fig. 2 and S2. $\dagger$ It can be clearly observed that the morphology of the oxidized SPCN/PANI- $X$ changes significantly with increase of the PANI content. Furthermore, the PANI nanorods exhibit a nanometer scale of about $50 \mathrm{~nm}$ in width and about $100-300 \mathrm{~nm}$ in length. When $0.005 \mathrm{M}$ of aniline monomer was added to the reaction system, the concentration of aniline monomer is too low to achieve uniform nucleation of the PANI nanorods on the oxidized SPCN. Therefore, the PANI nanorods are unevenly dispersed on the oxidized SPCN. This indicates that the lower concentration of aniline monomer cannot guarantee a wellcontrolled nucleation-growth condition for the PANI nanorods. However, the $0.01 \mathrm{M}$ aniline concentration can guarantee a well-controlled nucleation-growth condition for the PANI nanorods on the oxidized SPCN. So that PANI nanorods can be uniformly grown on the oxidized SPCN and even form a random network structure morphology. When the aniline monomer is increased to $0.02 \mathrm{M}$, the PANI nanorods are randomly and uniformly stacked on the oxidized SPCN. Obviously, there are many stacked pores on the stacked nanosheets. When the aniline monomer is further increased to $0.04 \mathrm{M}$, the PANI nanorods also exhibit a stacked morphology with a large number of stacked pore structures. These indicate that the oxidized SPCN is an effective 2D substrate material of the PANI nanorods. The PANI nanorods can be uniformly nucleated on their surface and eventually achieves uniform random accumulation on their surface. The aggregation morphology of the PANI nanorods on the oxidized SPCN is mainly controlled by the amount of aniline monomers. When $0.02 \mathrm{M}$ of aniline monomer was added to the reaction system, the uniformly stacked morphology of the PANI nanorods can be formed on the oxidized SPCN. When the amount of the aniline monomer was increased from $0.005 \mathrm{M}$ to $0.04 \mathrm{M}$, the content of the PANI nanorods in the oxidized SPCN/PANI electrode material was increased from $12.8 \%$ to $65.3 \%$ (Table S2 $\dagger$ ).

The porosity structure of the SPCN, oxidized SPCN, oxidized SPCN/PANI-0.02 and oxidized SPCN/PANI-0.04 were further evaluated by the $\mathrm{N}_{2}$ absorption/desorption isothermal analysis. The adsorption-desorption isotherm and the NLDFT pore size distribution are shown in Fig. 3a and b, and the relevant data are summarized in Table S3.† It is obvious that SPCN and 


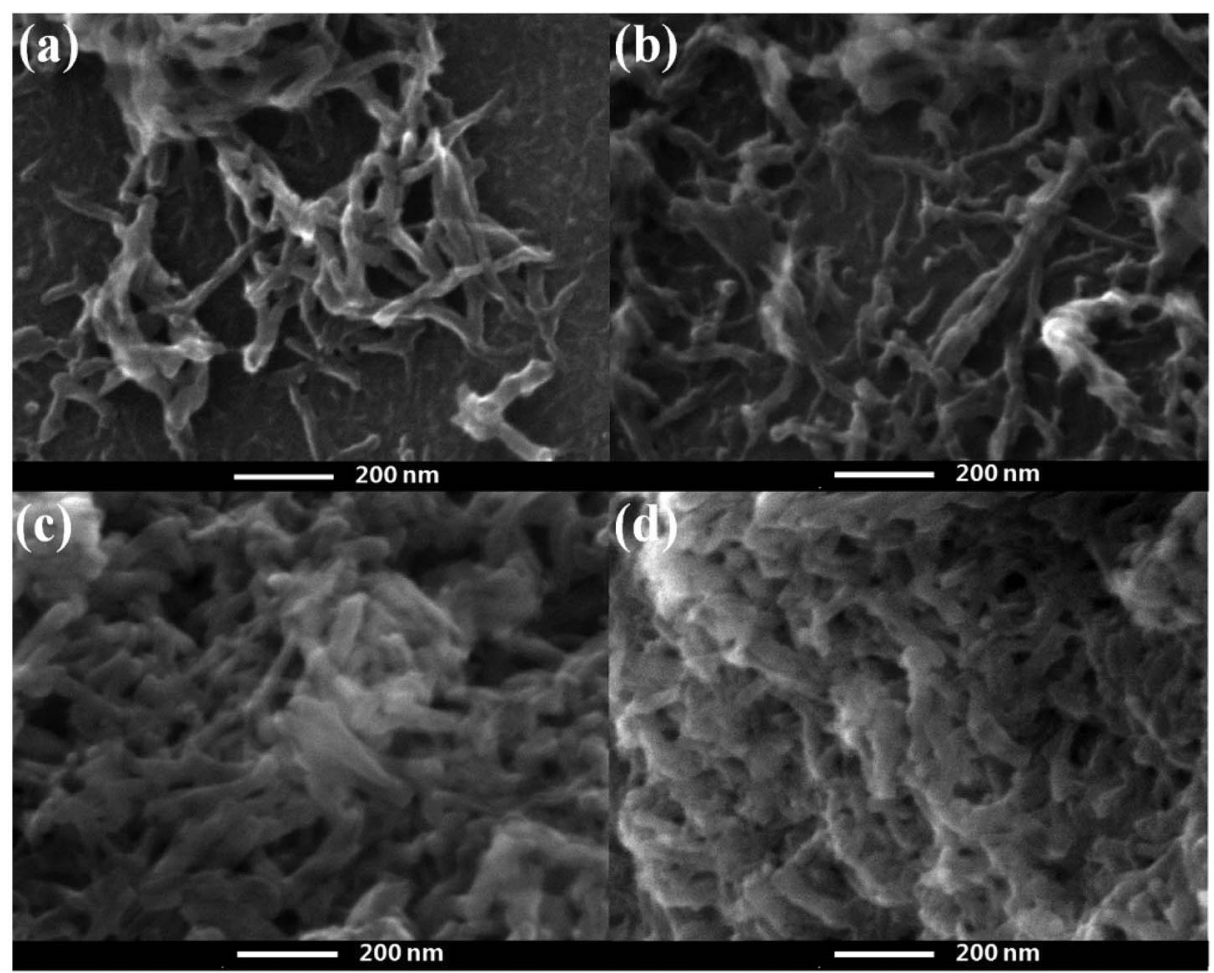

Fig. 2 FE-SEM of the oxidized SPCN/PANI-0.005 (a), the oxidized SPCN/PANI-0.01 (b), the oxidized SPCN/PANI-0.02 (c) and the oxidized SPCN/PANI-0.04 (d)

oxidized SPCN exhibit a type-I adsorption/desorption isotherm, which mean the SPCN and oxidized SPCN mainly possess micropore. The micropore of the SPCN is mainly present in the range of $0.37-1.31 \mathrm{~nm}$. After oxidation treatment, the micropore of the oxidized SPCN is mainly present in the range of $0.37-$
$0.52 \mathrm{~nm}$ and 1.24-1.48 nm. Furthermore, the BrunauerEmmett-Teller (BET) surface area is also reduced from $417 \mathrm{~m}^{2}$ $\mathrm{g}^{-1}$ of SPCN to $127 \mathrm{~m}^{2} \mathrm{~g}^{-1}$ of oxidized SPCN. These indicate that the oxidation treatment has a significant effect on the specific surface area and pore size distribution of the SPCN. The (a)
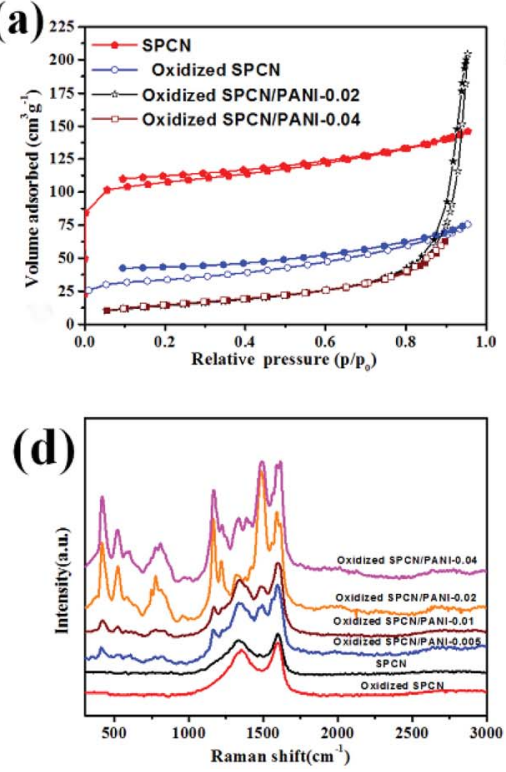
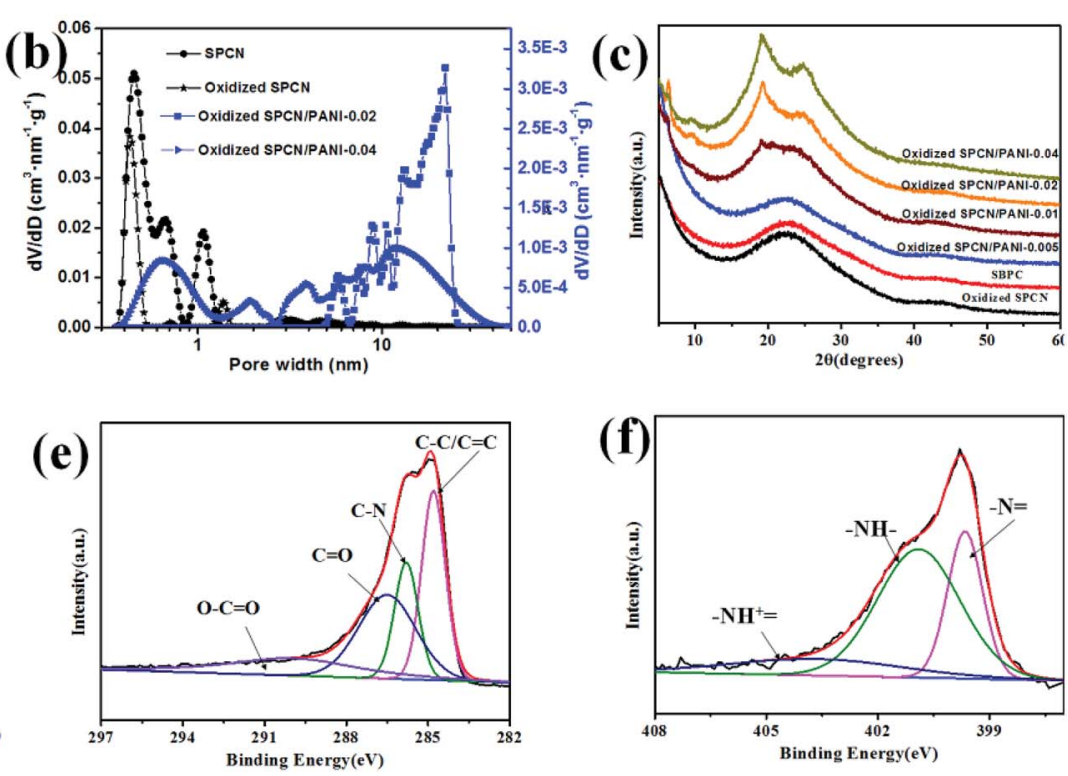

Fig. 3 Nitrogen adsorption/desorption isotherms (a), NLDFT pore distributions (b) of the SPCN, oxidized SPCN, oxidized SPCN/PANI-0.02 and oxidized SPCN/PANI-0.04; XRD patterns (c) and Raman spectra (d) of the SPCN, oxidized SPCN, oxidized SPCN/PANI-X; high resolution C 1s (e) and $\mathrm{N}$ 1s $(\mathrm{f})$ XPS spectra of the oxidized SPCN/PANI-0.02. 
adsorption-desorption isotherm of the oxidized SPCN/PANI0.02 presents a type-III adsorption/desorption isotherm, which mean the oxidized SPCN/PANI-0.02 mainly possess macropore. This is consistent with the results of the FE-SEM. The calculated BET surface area of the oxidized SPCN/PANI-0.02 and the oxidized SPCN/PANI-0.04 is about 52 and $53 \mathrm{~m}^{2} \mathrm{~g}^{-1}$. Furthermore, the NLDFT pore size distribution indicates that there is also a certain mesopores in the oxidized SPCN/PANI-0.02 and the oxidized SPCN/PANI-0.04. The increase in the amount of polyaniline nanorods seems to help the formation of micropores. These mesopores and macropores are mainly derived from random stack of the PANI nanorods on the oxidized SPCN, which not only facilitate the diffusion of ions in the stacked layer of the PANI nanorods, but also mitigate mechanical deformation of the PANI nanorods during the doping/dedoping process.

The structure of the SPCN, oxidized SPCN and the oxidized SPCN/PANI- $X$ was examined by XRD analysis (Fig. 3c). A broad diffraction peak (at around $2 \theta=22.7^{\circ}$ ) and a weak diffraction peak (at around $2 \theta=42.8^{\circ}$ ) indicates that the SPCN belongs to amorphous carbon. ${ }^{30}$ There is no significant change in the diffraction peak patterns of oxidized SPCN after oxidation treatment. This indicates that the oxidation treatment mainly only changes the surface chemical environment of the SPCN, and does not change the crystal structure of the oxidized SPCN. The characteristic diffraction peaks of the PANI nanowires gradually enhanced. The sharp peak at $2 \theta=6.3^{\circ}$ (indicates a short range ordering between the dopant and $\mathrm{N}$ atom on adjacent main chains) and two other broad peaks centered at $2 \theta$ $=19.3^{\circ}$ and $25.1^{\circ} .^{31,32}$ The structure of the SPCN, oxidized SPCN and the oxidized SPCN/PANI- $X$ was further estimated by Raman spectra (Fig. 3d). The Raman spectrum of the SPCN and oxidized SPCN displays a typical D band at $1338 \mathrm{~cm}^{-1}$ and $\mathrm{G}$ band at $1597 \mathrm{~cm}^{-1}$. For the oxidized SPCN/PANI- $X$, the characteristic peaks at $417,524,777,1163,1217,1378,1485$, and $1590 \mathrm{~cm}^{-1}$ are attributed to out-of-plane $\mathrm{C}-\mathrm{H}$ wag, out-of-plane $\mathrm{C}-\mathrm{N}-\mathrm{C}$ torsion, imine deformation, in-plane $\mathrm{C}-\mathrm{H}$ bending in emeraldine, $\mathrm{C}-\mathrm{N}$ stretching in emeraldine base (amine sites), $\mathrm{C}-\mathrm{N}^{+}$stretching in polaronic form (polarons), $\mathrm{C}-\mathrm{N}$ stretching in emeraldine base quinoid type ring (imine sites), and $\mathrm{C}=\mathrm{C}$ stretching in quinoid type ring, respectively, ${ }^{33,34}$ which indicates the presence of PANI in the oxidized SPCN/PANI- $X$.

X-ray photoelectron spectroscopy (XPS) analyse was further investigated to characterize the composition and chemical state of the oxidized SPCN/PANI-0.02 (Fig. 3e, f and S3 $\dagger$ ). The oxidized SPCN/PANI-0.02 shows a pronounced N 1 s peak at $400.2 \mathrm{eV}$. Elemental analysis by XPS reveals that $\mathrm{N}$ atom content of the oxidized SPCN/PANI-0.02 is about $11.6 \%$. The high-resolution C 1s spectrum can be fitted by four peaks located at around $284.8 \mathrm{eV}(\mathrm{C}-\mathrm{C} / \mathrm{C}=\mathrm{C}), 285.7 \mathrm{eV}(\mathrm{C}-\mathrm{N}), 286.4 \mathrm{eV}(\mathrm{C}=\mathrm{O})$, and $290 \mathrm{eV}(\mathrm{O}-\mathrm{C}=\mathrm{O})$. The high-resolution $\mathrm{N} 1 \mathrm{~s}$ spectrum can be deconvoluted into three peaks: quinoid imine $(-\mathrm{N}=$, centred at $399.6 \mathrm{eV})$, benzenoid amine (-NH-, centred at $400.9 \mathrm{eV})$ and positively charged nitrogen $\left(-\mathrm{NH}^{+}=\right.$, centred at $\left.403.7 \mathrm{eV}\right)$, indicating the successful random deposition of PANI nanorods on the oxidized SPCN.
Based on the unique morphology and the hierarchical pore structure, the as-obtained oxidized SPCN/PANI- $X$ is expected to be the promising candidate electrode material for supercapacitors. The electrochemical performance of the oxidized SPCN/PANI- $X$ was evaluated in a three-electrode system within $1 \mathrm{M} \mathrm{H}_{2} \mathrm{SO}_{4}$ electrolyte at room temperature. The cyclic voltammetry $(\mathrm{CV})$ curves of the oxidized SPCN/PANI- $X$ at a scan rate of $5 \mathrm{mV} \mathrm{s}^{-1}$ are shown in Fig. 4a. As can be seen, the CV curve of oxidized SPCN exhibits a quasi-symmetric rectangular shape, which indicates that the capacitance of oxidized SPCN is mainly derived from the electric double layer capacitance. However, the CV curves of the oxidized SPCN/PANI- $X$ demonstrate quasirectangular shapes, indicating the coexistence of pseudocapacitance and the electrical double-layer capacitance. The pseudocapacitance can be attributed to the transformation between leucoemeraldine base states and emeraldine salt states of PANI, and the transformation between emeraldine salt states and pernigraniline base states. ${ }^{35}$ Furthermore, the area enclosed by $\mathrm{CV}$ curve gradually increases with the amount of the aniline monomer increased from $0.005 \mathrm{M}$ to $0.02 \mathrm{M}$. However, when the amount of the aniline monomer is further increased up to $0.04 \mathrm{M}$, the area enclosed by $\mathrm{CV}$ curve becomes smaller. This phenomenon indicates that the oxidized SPCN/PANI-0.02 has the highest specific capacitance. The galvanostatic chargedischarge (GCD) curves of the oxidized SPCN and the oxidized SPCN/PANI- $X$ at a current density of $0.25 \mathrm{~A} \mathrm{~g}^{-1}$ are shown in the Fig. $4 \mathrm{~b}$. The GCD profile of the oxidized SPCN exhibits the typical isosceles triangle shape (the coulomb efficiency is about 1.0). This indicates that the capacitance of the oxidized SPCN mainly comes from the electrical double layer capacitive behavior. However, The GCD profiles of the oxidized SPCN/ PANI- $X$ exhibit the feature of pseudocapacitance (the curved charge/discharge lines. The coulomb efficiency of the oxidized SPCN/PANI-0.005, the oxidized SPCN/PANI-0.01, the oxidized SPCN/PANI-0.02, and the oxidized SPCN/PANI-0.04 are 0.8, 0.7, 0.6 and 0.6 , respectively). The discharging time gradually increases with the amount of the aniline monomer increased from $0.005 \mathrm{M}$ to $0.02 \mathrm{M}$. However, when the amount of the aniline monomer is further increased up to $0.04 \mathrm{M}$, the discharging time becomes shorter. The longest discharge time indicates that the oxidized SPCN/PANI-0.02 has the highest specific capacitance, which is well consistent with the CV results. The calculated specific capacitances of the oxidized SPCN/PANI- $X$ at the different current density are shown in the Fig. 4c. The specific capacitance values of the oxidized SPCN, the oxidized SPCN/PANI-0.005, the oxidized SPCN/PANI-0.01, the oxidized SPCN/PANI-0.02, and the oxidized SPCN/PANI0.04 are $185,334,378,513$, and $511 \mathrm{~F} \mathrm{~g}^{-1}$, respectively. The comparison of specific capacitance with previous reports is summarized in Table S1.† Obviously, the PANI nanorods can significantly improve the electrochemical properties of the oxidized SPCN. The highest specific capacitance of the oxidized SPCN/PANI-0.02 is attributed to its unique nano-morphology and the abundant multi-scale stacked porosity structure, which can improve electrolyte ion accessible surface area and provide the shortest electrolyte ion-diffusion path. When the current density is increased to $5 \mathrm{~A} \mathrm{~g}^{-1}$, the oxidized SPCN/PANI- 


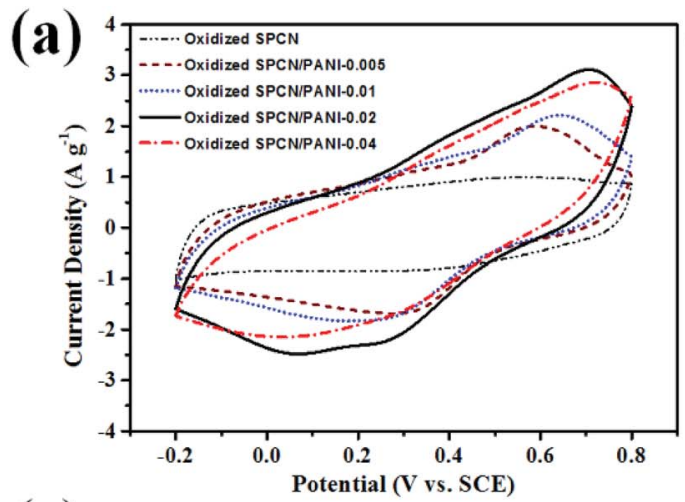

(c)

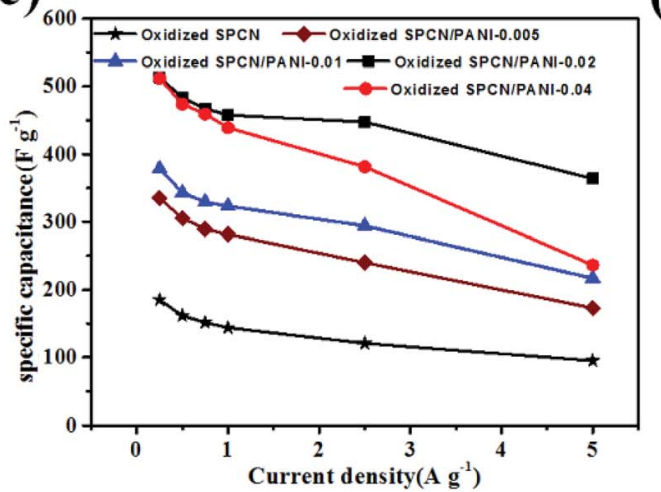

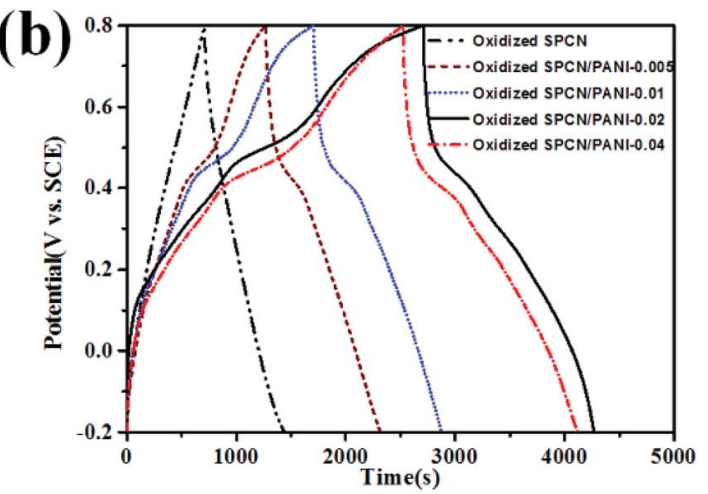

(d)

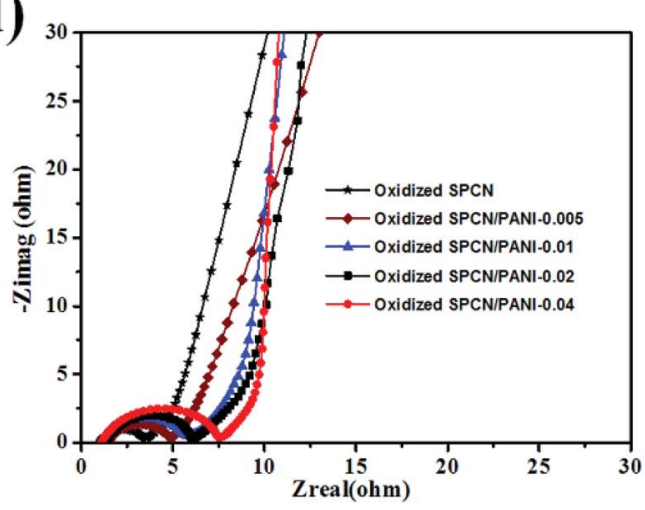

Fig. 4 (a) CV curves at the scan rate of $5 \mathrm{mV} \mathrm{s}^{-1}$ and (b) GCD curves at current density of $0.25 \mathrm{~A} \mathrm{~g}^{-1}$ of oxidized SPCN and oxidized SPCN/PANI-X; (c) specific capacitance versus current density and (d) Nyquist plots of oxidized SPCN and oxidized SPCN/PANI-X.

0.005, the oxidized SPCN/PANI-0.01, the oxidized SPCN/PANI0.02 , and the oxidized SPCN/PANI-0.04 retains a specific capacitance of $95,173,217,363$, and $236 \mathrm{~F} \mathrm{~g}^{-1}$, respectively, which is about $51 \%, 52 \%, 57 \%, 70 \%$, and $46 \%$ of the respective initial specific capacitance at $0.25 \mathrm{~A} \mathrm{~g}^{-1}$. The highest rate capability of the oxidized SPCN/PANI-0.02 indicates that the multi-scale stacked porosity structure exhibit efficient ion transport channels and electron transport path.

The ion diffusion and charge transfer kinetics of the oxidized SPCN/PANI- $X$ electrode material were further investigated by electrochemical impedance spectroscopy (EIS, Fig. 4d). Nyquist impedance plots of the oxidized SPCN and the oxidized SPCN/ PANI- $X$ exhibit almost the same profile. In the high frequency region, the equivalent series resistance (ESR, the interception of the Nyquist plots with the real axis) of the oxidized, the oxidized SPCN/PANI-0.005, the oxidized SPCN/PANI-0.01, the oxidized SPCN/PANI-0.02, and the oxidized SPCN/PANI-0.04 are 1.03, $1.50,1.06,1.30$, and $1.09 \Omega$, respectively. The low ESR value suggests that the random stacked morphology of the PANI nanorods still have good electric conductivity. The diameter of semicircle in the EIS spectrum gradually increases with the amount of the PANI nanorods increased from $12.8 \%$ to $65.3 \%$. Generally, the larger the diameter of the semicircle exhibits the higher charge transfer resistance $\left(R_{\mathrm{ct}}\right)$. Therefore, the more PANI nanorods randomly stacked on the oxidized SPCN exhibit the greater the transfer resistance. At the intermediate frequency region, Nyquist plots exhibit Warburg impedance (with a slope of the $45^{\circ}$ ). The projected length of Warburg impedance on the real axis can be used to evaluate the electrolyte ion reversibly diffusion process in porous electrode materials. ${ }^{36}$ The projected length does not become longer as the amount of the randomly stacked PANI nanorods increases. This indicates that a large number of stacked pores generated from random stacking of the PANI nanorods are beneficial to the rapid diffusion of electrolyte ions.

In order to evaluate real application of the oxidized SPCN/ PANI-0.02 electrode material, we assembled and investigated the oxidized SPCN/PANI-0.02 symmetric supercapacitors in the $1 \mathrm{M} \mathrm{H}_{2} \mathrm{SO}_{4}$ electrolyte. The CV curves (at different scan rates) and the charge-discharge curves (at different current densities) are shown in Fig. 5a and b. The specific capacitance of the oxidized SPCN/PANI-0.02-based symmetric supercapacitor at $0.25 \mathrm{~A} \mathrm{~g}^{-1}$ is $109 \mathrm{~F} \mathrm{~g}^{-1}$ (Fig. 5c. The coulomb efficiency is about $0.7)$. When the current density increases to $5 \mathrm{~A} \mathrm{~g}^{-1}$, the specific capacitance can still be maintained at $71 \%\left(78 \mathrm{~F} \mathrm{~g}^{-1}\right)$. The Ragone plot of the oxidized SPCN/PANI-0.02 is shown in Fig. 5d. The energy density of the oxidized SPCN/PANI- 0.02 reaches as high as $9.7 \mathrm{~W} \mathrm{~h} \mathrm{~kg}^{-1}$ at a power density of $50 \mathrm{~W} \mathrm{~kg}^{-1}$. When the power density increases to $1000 \mathrm{~W} \mathrm{~kg}^{-1}$, the energy density can still retain a value of $6.9 \mathrm{~W} \mathrm{~h} \mathrm{~kg}^{-1}$. The oxidized SPCN/PANI-0.02 also exhibits good cycling stability. The capacitance retention is still beyond $73.8 \%$ after 10000 charge-discharge cycles at $5 \mathrm{~A} \mathrm{~g}^{-1}$ (Fig. S5 $\dagger$ ). This indicates that the random stacked porous morphology can effectively mitigate mechanical 
(a)

formation of the PANI nanorods during the doping/dedoping process, and thus significantly improving the cycle stability.

\section{Conclusions}

In this article, sugarcane pith was selected as the self-template of $2 \mathrm{D}$ carbon nanosheets. And then the PANI nanorods were randomly deposited on the oxidized SPCN by using the dilute polymerization method. The random stacked morphology of the PANI nanorods on the oxidized SPCN can be effectively controlled by simply changing the molar mass of aniline monomer. When the molar mass of the aniline monomer is increased to $0.02 \mathrm{M}$, the PANI nanorods can be randomly and uniformly stacked on the oxidized SPCN. There are a large number of macropore and mesopore (5-26 nm) in the random stack layer of the PANI nanorods, which not only facilitate the diffusion of ions in the stacked layer of the PANI nanorods, but also mitigate mechanical deformation of the PANI nanorods during the doping/dedoping process. The oxidized SPCN/PANI0.02 exhibits the best electrochemical performance in $1 \mathrm{M}$ $\mathrm{H}_{2} \mathrm{SO}_{4}$. The largest specific electrode capacitance is up to $513 \mathrm{~F}$ $\mathrm{g}^{-1}$ at a current density of $0.25 \mathrm{~A} \mathrm{~g}^{-1}$. The oxidized SPCN/PANI0.02 also exhibits excellent electrochemical cycling stability.

\section{Conflicts of interest}

There are no conflicts to declare. plot of the oxidized SPCN/PANI-0.02 based symmetric supercapacitor. (b)

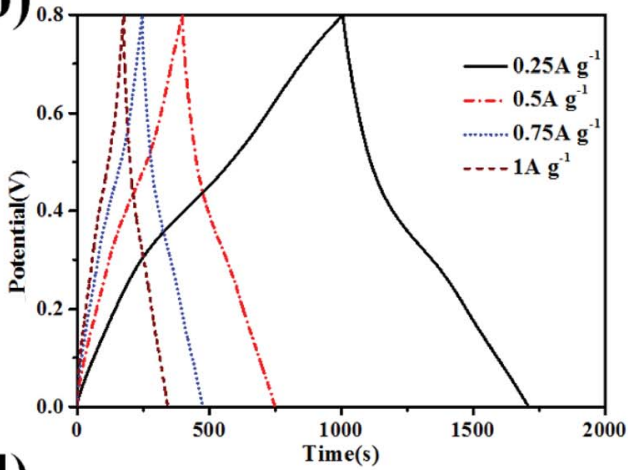

(d)

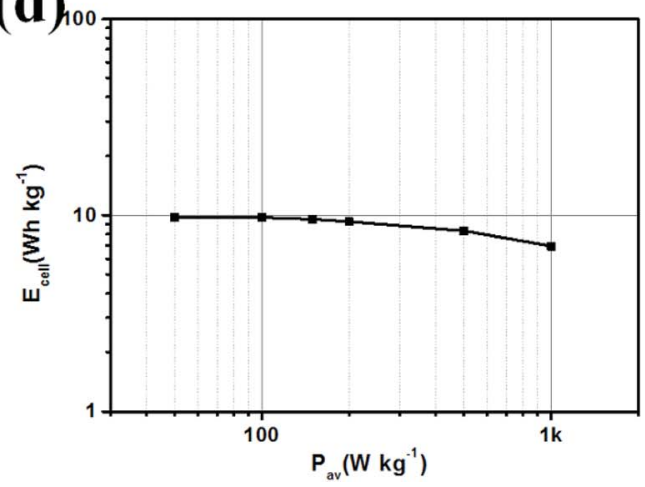

Fig. 5 (a) CV curves of the oxidized SPCN/PANI-0.02 based symmetric supercapacitor at different scan rates; (b) GCD curves of the oxidized SPCN/PANI-0.02 based symmetric supercapacitor at different current densities; (c) specific capacitance versus current density and (d) Ragone

\section{Acknowledgements}

Financial support was kindly supplied by grants from National Natural Science Foundation of China (No. 21601162, No. 21501154, and No. 21471135)

\section{References}

1 Y.-G. Wang, H.-Q. Li and Y.-Y. Xia, Adv. Mater., 2006, 18, 2619-+.

2 H. Talbi, P. E. Just and L. H. Dao, J. Appl. Electrochem., 2003, 33, 465-473.

3 E. Frackowiak, V. Khomenko, K. Jurewicz, K. Lota and F. Beguin, J. Power Sources, 2006, 153, 413-418.

4 A. Eftekhari, L. Li and Y. Yang, J. Power Sources, 2017, 347, 86-107.

5 G. Ciric-Marjanovic, Synth. Met., 2013, 177, 1-47.

6 H. Li, J. Wang, Q. Chu, Z. Wang, F. Zhang and S. Wang, J. Power Sources, 2009, 190, 578-586.

7 S. He, X. Hu, S. Chen, H. Hu, M. Hanif and H. Hou, J. Mater. Chem., 2012, 22, 5114-5120.

8 P. Yu, X. Zhao, Y. Li and Q. Zhang, Appl. Surf. Sci., 2017, 393, 37-45.

9 J. Wu, Q. e. Zhang, J. Wang, X. Huang and H. Bai, Energy Environ. Sci., 2018, 11, 1280-1286.

10 N. A. Kumar, H.-J. Choi, Y. R. Shin, D. W. Chang, L. Dai and J.-B. Baek, ACS Nano, 2012, 6, 1715-1723. 
11 X. Li, C. Zhang, S. Xin, Z. Yang, Y. Li, D. Zhang and P. Yao, ACS Appl. Mater. Interfaces, 2016, 8, 21373-21380.

12 D.-W. Wang, F. Li, J. Zhao, W. Ren, Z.-G. Chen, J. Tan, Z.-S. Wu, I. Gentle, G. Q. Lu and H.-M. Cheng, ACS Nano, 2009, 3, 1745-1752.

13 N.-R. Chiou, C. Lui, J. Guan, L. J. Lee and A. J. Epstein, Nat. Nanotechnol., 2007, 2, 354-357.

14 N. R. Chiou and A. J. Epstein, Adv. Mater., 2005, 17, 16791683.

15 C. Xia, W. Chen, X. Wang, M. N. Hedhili, N. Wei and H. N. Alshareef, Adv. Energy Mater., 2015, 5, 1401805.

16 C. O. Baker, X. Huang, W. Nelson and R. B. Kaner, Chem. Soc. Rev., 2017, 46, 1510-1525.

17 R. Malik, L. Zhang, C. McConnell, M. Schott, Y.-Y. Hsieh, R. Noga, N. T. Alvarez and V. Shanov, Carbon, 2017, 116, 579-590.

18 N. Hui, F. Chai, P. Lin, Z. Song, X. Sun, Y. Li, S. Niu and X. Luo, Electrochim. Acta, 2016, 199, 234-241.

19 X. Hong, B. Zhang, E. Murphy, J. Zou and F. Kim, J. Power Sources, 2017, 343, 60-66.

20 Y. Meng, K. Wang, Y. Zhang and Z. Wei, Adv. Mater., 2013, 25, 6985-6990.

21 K. Wang, Q. Meng, Y. Zhang, Z. Wei and M. Miao, Adv. Mater., 2013, 25, 1494-1498.

22 J. Xu, K. Wang, S.-Z. Zu, B.-H. Han and Z. Wei, ACS Nano, 2010, 4, 5019-5026.

23 Z.-Y. Wu, H.-W. Liang, L.-F. Chen, B.-C. Hu and S.-H. Yu, Acc. Chem. Res., 2016, 49, 96-105.

24 Z.-Y. Wu, C. Li, H.-W. Liang, J.-F. Chen and S.-H. Yu, Angew. Chem., Int. Ed., 2013, 52, 2925-2929.
25 D.-L. Huang, R.-Z. Wang, Y.-G. Liu, G.-M. Zeng, C. Lai, P. Xu, B.-A. Lu, J.-J. Xu, C. Wang and C. Huang, Environ. Sci. Pollut. Res., 2015, 22, 963-977.

26 J. Hou, C. Cao, F. Idrees and X. Ma, ACS Nano, 2015, 9, 25562564.

27 L.-F. Chen, Z.-H. Huang, H.-W. Liang, W.-T. Yao, Z.-Y. Yu and S.-H. Yu, Energy Environ. Sci., 2013, 6, 3331-3338.

28 S.-C. Li, B.-C. Hu, Y.-W. Ding, H.-W. Liang, C. Li, Z.-Y. Yu, Z.-Y. Wu, W.-S. Chen and S.-H. Yu, Angew. Chem., Int. Ed., 2018, 57, 7085-7090.

29 Q. Niu, K. Gao, Q. Tang, L. Wang, L. Han, H. Fang, Y. Zhang, S. Wang and L. Wang, Carbon, 2017, 123, 290-298.

30 Y. Xu, C. Zhang, M. Zhou, Q. Fu, C. Zhao, M. Wu and Y. Lei, Nat. Commun., 2018, 9, 1720.

31 S. Zhu, X. Chen, Y. Gou, Z. Zhou, M. Jiang, J. Lu and D. Hui, Polym. Adv. Technol., 2012, 23, 796-802.

32 X. Li, L. J. Yu, L. M. Yu, Y. B. Dong, Q. Gao, Q. X. Yang, W. T. Yang, Y. F. Zhu and Y. Q. Fu, Chem. Eng. J., 2018, 352, 745-755.

33 N. Hui, F. Chai, P. Lin, Z. Song, X. Sun, Y. Li, S. Niu and X. Luo, Electrochim. Acta, 2016, 199, 234-241.

34 Y. Guo, T. Wang, F. Chen, X. Sun, X. Li, Z. Yu, P. Wan and X. Chen, Nanoscale, 2016, 8, 12073-12080.

35 S.-X. Zhou, X.-Y. Tao, J. Ma, C.-H. Qu, Y. Zhou, L.-T. Guo, P.-Z. Feng, Y.-B. Zhu and X.-Y. Wei, Vacuum, 2017, 143, 6370.

36 H. Koga, H. Tonomura, M. Nogi, K. Suganuma and Y. Nishina, Green Chem., 2016, 18, 1117-1124. 